\title{
Dynamique des populations larvaires de Baetis navasi M.-L. (Ephemeroptera) dans un torrent du Haut-Atlas de Marrakech, Maroc
}

\author{
H. Ouahsinel \\ P. Lavandier ${ }^{2}$
}

Muts clés : Insecte, Ephemeroptera, cycle biologique, croíssance mortalite, production.

Le cycle biologique de Baetis navasi dure un an dans un torrent de montagne du Maroc (altitude $2600 \mathrm{~m}$ ) - Température $0-22^{\circ} \mathrm{C}$. couvert de neige 1 mois par an). Les adultes volent en juin-juillet. Les larves se nourrissent de périphyton et vivent préférentiellement dans la végétation (Ranunculıs aquatilis). La croissance larvaire est exponentielle et les taux de croissance (en poids sec) sont de 1,74\% par jour de juillet à décembre, proches de zéro en hiver et de 1,46\% par jour au prin. temps avant le début des émergences. La mortalité, forte dans les 2 premiers mois du cycle $(4,9 \%$ par jour) est plus faible de septembre à mars (1,16\% par jour) et nulle au printemps. La production annuelle peut être estimée à $440 \mathrm{mg}$ de poids $\mathrm{sec}$ par $\mathrm{m}^{2}$. Le rapport production/Biomasse moyenne $=4,9$.

Larval population dynamics of Baetis navasi (Ephemeroptera) in a torrential river in the Haut-Atlas of Marrakech-Maroc

Keywords: Insect, ephemeroptera, life cycle, growth, mortality, production.

The life cycle of Baetis navasi took one year in a torrential river in Marocco (altitude : $2600 \mathrm{~m}$; temperature $0-22^{\circ} \mathrm{C}$ : snow-cover I month/year). Adults flew in June and July. Larvae feed on periphyton and lived chiefly in Ranumculus aquatilis. The larval growth was exponential and the mean growth rate was $1,74 \%$ day $^{-1}$ (dry weight) from July to December, close to zero in winter and $1,46 \%$ day $^{-1}$ in spring before the adults started to emerge. Mortality was high in the first two months of the cycle $(4,9 \%$ day -1$)$, lower from september to January $(1,16 \%$ day-1) and close to zero in spring. The annual production estimate was $440 \mathrm{mg}$ dry $\mathrm{wt}^{2} \mathrm{~m}^{2}$. The ratio of production to mean standing crop $(\mathbf{P} / \overline{\mathrm{B}})$ was 4,9 .

Baetis navasi Muller-Liebenau est un éphéméroptère connu de la péninsule ibérique - Terra typica : Portugal - (Puthz 1978, Alba Tercedor 1981) et largement répandu dans la partie nord de l'Afrique (A.G.B. Thomas, comm. pers.). A not re connaissance toutefois, il n'existe pas de données concernant la dynamique des populat ions de cette espèce. Le présent travail décrit le cycle biologique de Baetis navasi pour la première fois: il précise la croissance, la mortalité des larves et aborde quelques aspects de leur biologie ; au travers des estimations

1. Université Cadi Ayyad, Faculté des Sciences, Boulevard de Safi, BP. 515 Marrakech, Maroc.

2. Laboratoire d'Hydrobiologie, U.A. CNRS 695, Université Paul Sabatier, 31062 Toulouse Cédex, France. de biomasse et de production, il quantifie l'importance de l'espèce au sein de la biocénose d'un torrent marocain de haute montagne.

\section{Milieu - Matériel - Méthodes}

Le ruisseau de Tiferguine est un torrent permanent du réseau hydrographique de l'Ourika (HautAtlas de Marrakech, Maroc). Long de 2,5 km, il prend sa source à $2700 \mathrm{~m}$ d'altitude et traverse des terrains essentiellement cristallins couverts de pelouses et de xérophy tes épineux avant de se jeter dans le ruisseau d'Oukaimeden à $2500 \mathrm{~m}$ d'altitude.

A la station d'étude $(2600 \mathrm{~m}, 1 \mathrm{~km}$ de la source) le lit est étroit, essentiellement pierreux et couvert 


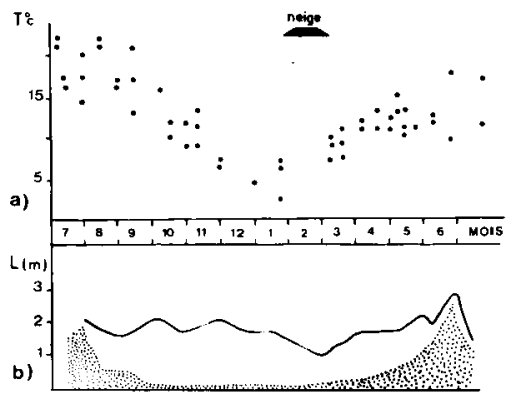

Fig. 1 : Torrent de Tiferguine a) températures ponctuelles relevées à chaque date d'échantillonnage. b) évolution de la largeur (L) du cours et importance de la végétation (en pointillés) au long de l'année.

d'une végétation dense de Ranunculus aquatilis et de Roriparia nasturtium dont l'importance fluctue au cours de l'année (fig. lb).

Plusieurs mesures instantanées de température ont èté faites à chaque date d'échantillonnage (fig. 1a). Les plus fortes valeurs sont très voisines des maximas journaliers. Les minimas sont proches de $0^{\circ} \mathrm{C}$ deux mois par an (fin décembre-mi février). Le torrent peut disparaitre totalement sous la neige en février.

Les débits qui dépendent de la pluviosité et de la fonte des neiges sont maximums au printemps $(>$ $\left.2501 \mathrm{sec}^{-1}\right)$, minimums en début d'hiver $(<201$ sec $^{-1}$ (Pihan \& Mohati 1983). Au cours de l'année. les vitesses de courant varient de 2 à $30 \mathrm{~cm} \mathrm{sec}-1$ dans les zones calmes et de 20 à $100 \mathrm{~cm} \mathrm{sec}^{-1}$ dans les zones à courant rapide.

La largeur moyenne du cours d'eau présente d'importantes variations liées à la fois aux fluctuations de débit et à la densité de la végétation qui ralentit l'écou lement ; elle est estimée à chaque échantillonnage (fig. $1 \mathrm{~b}$ ).

La minéralisation est faible (de l'ordre de $80,1 \mathrm{~s} \mathrm{~cm}-1$ ) due à la nature cristalline du substrat. Le $\mathrm{pH}$, légèrement basique en hiver, peut dépasser 9,5 en juin-juillet lorsque la végétation est abondante.
Plus de 13000 larves ont été capturées à l'aide d'un filet de Surber (surface prélevee $1 / 10 \mathrm{~m}^{2}$. vide de maille $0,3 \mathrm{~mm}$ ). Trois à six relevés ont été faits 1 ou 2 fois par mois dans les divers habitats de la station entre juillet 1985 et juillet 1986. La faune est fixee au formol, puis conservée en alcool. Les adultes sont capturés par chasse et la période de vol est précisée par la présence de larves au dernier stade prêtes à émerger.

La largeur des capsules céphaliques ( $\mathrm{kc}$ ) a été utilisée comme critère de taille. On peut en déduire la longueur $L$, le poids frais PF el le poids sec PS (après dessèchement à l'étuve à $60^{\circ}$ pendant $24 \mathrm{~h}$ ) des larves par les relations suivantes:

$\mathrm{L} \mathrm{mm}=7,356 \mathrm{lcc}+0,43$

$\log$ PF $\mathrm{mg}=3,38 \log \mathrm{lcc}(\mathrm{mm})+1,888$

$\log$ PS $m g=3,136 \log$ lcc $(m m)+0,147$

A chaque date d'échantillonnage, le nombre moyen d'individus par $\mathrm{m}^{2}$ de torrent est calculé à partir des densités observées dans chaque type de substrat et de l'importance relative des différents substrats dans le milieu. Afin d'être comparables entre elles, les estimations doivent en outre tenir compte des importantes variations de largeur du cours d'eau puisque la faune se concentre ou se disperse en fonction de la surface immergée (Lavandier 1979). Pour cela, nous avons exprimé les résul tats (densité. biomasse, production) par rapport a la plus grande largeur observée durant l'étude ( $3 \mathrm{~m}$. le 24/06/86). Ainsi par exemple, la densité de $B$. navasi qui était de 640 individus $m^{-2}$ dans un cours d'eau large de $1,75 \mathrm{~m}$ le $29 / 10 / 85$ a ćté ramenée à 373 individus $\mathrm{m}^{-2}\left(640 \times \frac{1.75}{3}\right)$, c'est-à-dire à la densité que l'on aurait théoriquement rencontrée si la largeur était restée égale a $3 \mathrm{~m}$.

\section{Résultats}

\subsection{Répartition}

Baetis navasi peuple tous les biotopes mais à surface comparable, les végétaux abritent en moyenne 6 à 7 fois plus d'individus que les substrats minéraux (fig. 2). Ce préférendum est constant et indépendant de l'âge des larves et des végétaux au cours du cycle annuel. L'importance des populations larvaires est donc étroitement liée au développement de la végétation aquatique qui peut couvrir au début de l'été les 9/10 de la surface immergée.

\subsection{Régime alimentaire}

Une quarantaine de larves de tailles variées ont été disséquées à six périodes de l'année. Quelle que 


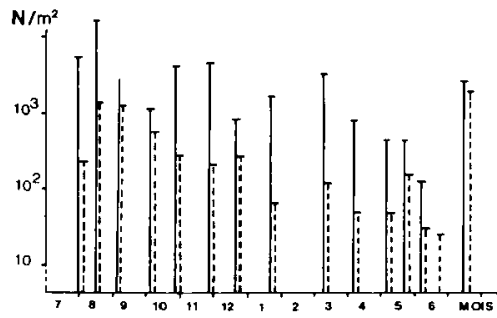

Fig. 2 : Répartition de Baetis navasi : nombre d'individus ( $\mathrm{N} / \mathrm{m}^{2}$ ) dans la végétation (traits continus) et sur substrat de pierres, cailloux, graviers (tirets).

soit leur taille, les contenus intestinaux renferment en permanence de nombreuses algues périphytiques avec notamment des diatomées telles Diatoma hiemale, var. mesodon, Synedra ulna, Meridion circulare, Achnantes minutissima, Navicula sp., Gomphonema parvulum, Fragilaria intermedia, Cocconeis placentula et des cyanophycées Nostoc sp., Oscillatoria sp. La présence de débris ligneux et la rareté des particules minérales montrent que le broutage s'effectue essentiellement sur les macrophytes.

\subsection{Dynamique du peuplement}

Le cycle biologique est déduit de la structure des peuplements à chaque date d'échantillonnage ( $f i g$. 3 ) ; l'évolution du poids sec individuel moyen des larves et du nombre d'individus par $\mathrm{m}^{2}$ peuvent être assimilées à des portions de courbes exponentielles en fonction du temps ( $f \mathrm{ig}$. 4). Excepté pour les périodes décembre-mars en ce qui concerne la croissance pondérale et janvier-mai en ce qui conceme l'évolution numérique, il existe une relation significative ou hautement significative entre les Logarithmes des poids (ou des nombres d'individus) et le temps.

\section{CyCle biologioue et CROISSANCE laRVAIRE}

La période de vol, déduite de la présence de lar. ves au dernier stade aux fourreaux alaires noirs est courte : fin mai - fin juin. Les éclosions se produisent en juillet-août et rapidement plusieurs classes de taille sont simultanément présentes dans le milieu. Les premières ébauches de fourreaux alaires sont visibles en octobre chez les larves les plus âgées. Durant cette période, le taux de croissance ( \pm Lc $95 \%$ ) du poids sec individuel moyen des larves est de 1,7 $\pm 0,49 \%$ par jour. La croissance est ensuite très lente $(0,15 \%$ par jour mais relation non significative) au cours de l'hiver ; elle reprend dès le mois de mars $(1,15 \pm 0,4 \%$ par jour $)$ jusqu'au début des émergences ; les derniers stades larvaires apparaissent en avril et le développement s'achève en mai. L'espèce est univoltine, avec des périodes de vol et d'éclosion courtes et une croissance homogène de la majorité des larves.

\section{EVOLUTION NUMÉRIQUE}

Quatre phases se succèdent après l'augmentation des effectifs consécutive à l'éclosion des larvules ( $f$ ig. 4). Au début du développement, la mortalité est forte et le taux de diminution ( \pm Lc $95 \%$ ) peut être estimé à $4,9 \pm 1,2 \%$ par jour. De septembre à janvier, la mortalité est plus faible $(1,16 \pm 0,9 \%$ par jour $)$. $\mathrm{La}$ population demeure ensuite remarquablement stable jusqu'à la période d'émergence où $11 \%$ des larves disparaissent chaque jour.

\section{Biomasse et PRODUCTION}

La biomasse moyenne annuelle ét ablie d'après les biomasses moyennes mensuelles est de $90 \mathrm{mg}$ PS $m^{-2}$. Les plus fortes biomasses ( $200 \mathrm{mg}$ PS $m^{-2}$ ) s'observent au dćbut du cycle biologique avec des larves petites mais nombreuses et en fin de développement lorsque les larves sont peu nombreuses mais de grande taille.

La production larvaire qui intègre l'évolution numérique et pondérale de la population au cours du cycle vital a été calculée par la méthode graphique d'Allen et à partir des accroissements successifs de biomasse. Les deux méthodes donnent des résultats très voisins et la production peut être estimée à $440 \mathrm{mg} \mathrm{PS} \mathrm{m}^{-2} \mathrm{an}^{-1}$; elle s'effectue à part égale avant et après la période hivernale. Le rapport Production/Biomasse moyenne est de 4,9 .

\section{Discussion}

Le cycle biologique de Baetis navasi, décrit ici pour la première fois est typiquement annuel. L'unique génération se développe en 11 mois environ et présente l'alternance classique, mais particulièrement accusée en montagne (Lavandier \& Décamps 1984), de phases hivernales de repos et estivales de croissance. Du fait de la brièveté des périodes d'éclosion et d'émergence, les taux de croissance individuels moyens des larves estimés sur l'ensemble de la 


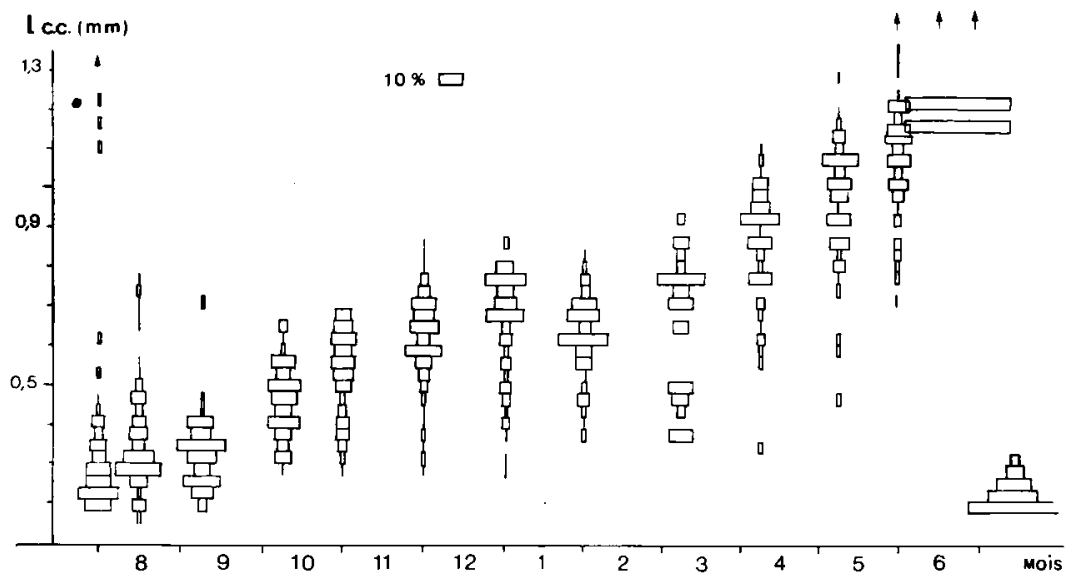

Fig. 3 : Cycle biologique de Baetis navasi : structure des populations larvaires (lcc = largeur des capsules céphaliques) эu cours de l'année. Les flèches indiquent les périodes d'émergence.

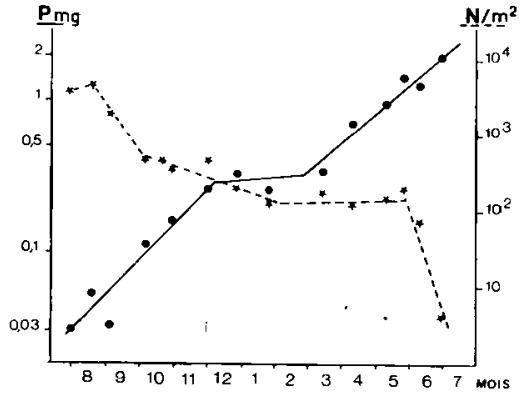

Fig. 4 : Dynamique des populations de Baetis navasi : évolution du poids individuel moyen (P/mg - traits continus) et de l'effectif des larves (N/m $/ \mathbf{m}^{2}$ tirets) au cours du cycle biologique. population se rapprochent vraisemblablement de ceux de cohortes bien individualisées au sens de Benech (1972) ou Humpesch (1979). Bien qu'ils ne soient pas significativement différents en dehors de la période hivernale, les taux de croissance paraissent néanmoins liés à la température moyennc du milieu que l'on peut estimer grossierement à partir des relevés ponctuels, étant donné les modalités de réchauffement de l'eau dans un torrent de montagne (Lavandier 1974) : 1,73\% $\mathrm{j}^{-1}$ pour une température moyenne de l'ordre de $11-13^{\circ} \mathrm{C}, 0,15 \% \mathrm{j}^{-1}$ pour $\mathrm{T}=4,5-6,5^{\circ} \mathrm{C}, 1,46 \% \mathrm{j}^{-1}$ pour $\mathrm{T}=9,5$ $11,5^{\circ} \mathrm{C}$. Compte tenu de la relation existant entre le poids sec et la longueur des larves (Log PS mg = 3,5 Log Lmm - 7), ces taux de croissance pondérale correspondent à des taux de croissance en longueur de $0,49 \% \mathrm{j}-1,0,043 \% \mathrm{j}-1$ et $0,42 \% \mathrm{j}^{-1}$ (taux de croissance en longueur $=$ taux de croissance pondérale/3,5). Ces taux de croissance sont parmi les plus faibles observés pour plusieurs espèces d'éphéméroptères à durée de vie voisine : Ephemera strigata (Gose 1970) Ephemerella subvaria (Waters \& Crowford 1973) Leplophlebia vesperina (Brittain 
1976) ou étudiées dans des caux plus froides Baetis alpinus (Lavandier 1988) ou B. bicaltalus (Pearson \& Kramer 1972). Ils sont également net tement inférieurs à ceux des Baetidés qui, dans des conditions de température grossièrement comparables ont un cycle vital plus court (cf. Humpesch 1979).

L'étude de l'évolution numérique est délicate à préciser car à l'hétérogénéité du milieu, s'ajoutent le's variations annuelles de la surface immergée. L'expression des résultats en fonction d'une surface invariable permet la comparaison des densités évaluées au cours de l'année. Elle sous-entend toutefois une migration rapide des larves en réponse aux variations de débit et nécessite à chaque échantillonnage d'estimer l'importance relative des différents substrats et de la surface mouillée. Malgré la difficulté des mesures sur le terrain, les résultats s'ordonnent de façon remarquable : après la mortalité classiquement forte des jeunes larves, la diminution des effectifs se ralentit puis s'annule au cours des 4 mois précédant l'émergence; début juin, on recueille encore plus de $3 \%$ de l'effectif maximum observé en début du cycle. Cette valeur de survie qui ne tient pas compte de la mortalité larvaire au tout début et en fin de cycle biologique est néanmoins élevée : elle souligne le rôle de la couverture végétale qui assure nourriture et refuge aux populations.

La biomasse moyenne $\left(90 \mathrm{mg} \mathrm{m}^{-2}\right)$ et la production ( $440 \mathrm{mg} \mathrm{m}^{-2}$ ) annuelles mont rent l'importance de l'espèce dans la biocénose. Le rapport Production/Biomasse voisin de 5 est très proche de la moyenne des valeurs obtenues pour les espèces univoltines (Waters 1977, Lavandier 1979).
Travaux cités

Alba Tercedor (J.) 1984 - Recopilación de citas de efemerópteros en la peninsula iberica e islas baleares. Trab. Monogr. Dep. Zool Univ Granada (N.S.) 4 (2): 41.81 .

Benech (V.). 1972. - Le polyvoltisme chez Baetis rhodani Pictet (Insecta, Ephemeroptera) dans un ruisseau à truites des Pyrénées-Atlantiquess, Le Lissuraga. Ann. Hydrobiol., 3: $141 \cdot 171$

Brittain (J.E.). 1976. - Experimental studies on nymphal growth in Leptophlebia vespertina (L.) (Ephemeroptera). Freshwat. Biol., 6 : 445.449

Gose (K.). 1970. - Life history and production rates of Ephemere srrigala (Ephemeroptera). Jap. J. Limnol., 31: $21-26$.

Humpesch (U.H.). 1979. - Life cycles and growth rates of Baetis spp. (Ephemeroplera : Baetidae) in the laboratory and in two stony streams in Austria. Freshwat. Biol., 9: 467-479.

Lavandier (P.). 1974. - Ecologie d'un torrent pyrénéen de haute montagne. I. Caractéristiques physiques. Annls. Limnol., 10 : 173-219.

Lavandier (P.). 1979. - Ecologie d'un torrent pyrénéen de haute montagne : l'Esta ragne. Thèse Doctorat d'Etat Sciences U.P.S. Toulouse : 532 p.

Lavandier (P.). 1988. - Semivoltinisme dans des populations de haute montagne de Baetis alpimus Pictet (Ephemeroptera). Bull. Soc: Hist. Nat.. Totslouse, $124: 61$-64.

Lavandier (P.) \& Decamps (H.). 1984 - Estaragne. in Ecology of European Rivers. Whitton B.A. ed. Blackwell Scientific Publj. cations. p. 237-264.

Pearson (W.D.) \& Kramer (R.H.). 1972. - Drift and production of two aquatic inscets in a mountain stream. Ecol. Monogr. 24 : 365-385.

Pihan (J.C.) \& Mohati (A.). 1983. - Elude hydrobiologique de deux petits torrents du Haut-Atlas de Marrakech. Biol. Fac. Sci. Mar rakech. $2: 23.42$.

Puthz (S.). 1978. - Ephemeroptera in Limnofauna europea, Illies J. ed. : 256-263.

Waters (T.F.) 1977. - Secondary Production in Inland Waters Adv, Ecol. Res., 10:91-164.

Waters (T.F.) \& Crowford (G.W.). 1973. - Annual production of a stream mayfly population : comparison of methods. Limnol. Oceanogr.. 18 : 286-296. 